\title{
Fin de la era Trump y las promesas de Biden: ¿nuevos aires en la política migratoria de la Cuarta Transformación?
}

\author{
End of the Trump era and the promises of Biden: new airs \\ in the migration policy of the Fourth Transformation?
}

Daniel Villafuerte Solís*

ISSN IMPRESO 1870-7599 | ISSN RED CÓMPUTO 2448-7783 | 103-127

María del Carmen García Aguilar**

RECIBIDO 17/11/20 | ACEPTADO 2/12/20

\begin{abstract}
Resumen. Este artículo analiza el cambio en la política migratoria del gobierno de México, misma que transitó de una propuesta de política integral a una política parcial y punitiva que profundiza el drama de los migrantes y sus familias. La hipótesis subyacente sostiene que, si bien el giro en el tratamiento de la migración centroamericana del actual gobierno de México se explica por la enorme presión del gobierno de Donald Trump, que llegó a la aplicación de medidas extremas materializadas en la política de tolerancia cero, el desequilibrio interestatal del poder hemisférico y regional impone límites a políticas que intenten subvertir la hegemonía que hoy ostenta Estados Unidos. Por ello resulta pertinente, a la luz de la crisis global y estadounidense, cuestionar la ruta de Joe Biden, que prometió enviar una iniciativa de ley migratoria y revertir la política seguida por Trump en esta materia.
\end{abstract}

Palabras clave: Cuarta Trasformación, política migratoria, frontera sur, tolerancia cero, Triángulo Norte.

\begin{abstract}
This article analyzes the change in the migration policy of the Mexican government, which went from a comprehensive policy proposal to a partial and punitive policy that intensifies the drama of migrants and their families. The underlying hypothesis holds that the shift in the treatment of Central American migration by the current government of Mexico is explained by the huge pressure of the Donald Trump government, which led to the application of extreme measures materialized in the zero tolerance policy, but the interstate imbalance of the hemispheric and regional power imposes limits on policies that attempt to subvert the hegemony that the United States now holds. For this reason, it is pertinent, in light of the global and US crisis, to question the route of Joe Biden, who promised to send an immigration law initiative and reverse the policy followed by Trump in this matter.
\end{abstract}

Keywords: Fourth Transformation, immigration policy, southern border, zero tolerance, Northern Triangle.

\footnotetext{
* Mexicano. Profesor investigador del Centro de Estudios Superiores de México y Centroamérica, de la Universidad de Ciencias y Artes de Chiapas. Correo-e: gasoda_2000@yahoo.com.mx

** Mexicana. Profesora investigadora del Centro de Estudios Superiores de México y Centroamérica, de la Universidad de Ciencias y Artes de Chiapas. Correo-e: mcgarcia2005@yahoo.com.mx
} 


\section{Introducción}

Desde el 11 de septiembre de 2001 el fenómeno migratorio centroamericano ha venido ocupando centralidad en la agenda de México y de Estados Unidos. A partir de entonces la obsesión de los gobiernos ha sido el «sellamiento de la frontera sur» de México. Uno de los primeros programas encaminados a este propósito fue el llamado Plan Sur, que se lanzó de manera paralela al Plan Puebla Panamá, al inicio de la administración del presidente Vicente Fox.

Hasta antes de la llegada de Donald Trump a la presidencia podría decirse que la política migratoria de tránsito por México era más o menos flexible. Esta flexibilidad se explica por el contubernio de las autoridades migratorias con las redes de tráfico de personas, y por la relativa apertura del gobierno de Estados Unidos a la entrada de mano de obra barata.

En el presente nos encontramos frente a una nueva época de las migraciones. Los orígenes de las migraciones se han ampliado, las dificultades en el mundo han crecido, la emergencia y agudización de las guerras, de los conflictos internos, la exclusión social y la destrucción de la sociedad basada en el trabajo (Gorz, 2000) configuran esta nueva época, marcada por la violencia y por un modelo de sociedad global centrada en el interés material.

La migración por razones laborales y el desplazamiento son las dos caras de la movilidad humana, ambas comparten la característica de ser «forzadas». El desplazamiento obedece a diversos factores, desde conflictos al interior y entre comunidades, hasta sequías prolongadas y guerras como ha sido la historia de Centroamérica hasta mediados de la década de 1980, y ahora con énfasis en el Oriente Medio y África, principalmente.

En este orden de ideas, el propósito del presente artículo es plantear la naturaleza del fenómeno de la movilidad humana centroamericana, así como analizar las medidas implementadas por el gobierno de la Cuarta Transformación que se expresan en la contención de los flujos irregulares. Estamos frente al fin de la administración de Trump y con ello resurge la esperanza de una política migratoria más tolerante; sin embargo, nos preguntamos sobre sus posibilidades en tanto que el trumpismo estará presente en las cámaras y en la sociedad, además de la emergencia de la covid-19 que se ha constituido en una aliada en el «sellamiento» de la frontera sur de México.

${ }^{1}$ En 2004, Stephen Castles y Mark J. Miller titularon a su libro La era de la migración. Movimientos internacionales de población en el mundo moderno. 


\section{Movilidad humana, caravanas y nueva política migratoria}

Desde las guerras en Centroamérica, el desplazamiento forzado y las migraciones por motivos económicos se constituyeron en un fenómeno que fue creciendo hasta convertirse en un componente estratégico de los medios de vida de miles de centroamericanos. De esta manera, se erigió un sistema migratorio donde las redes migratorias y lo que se ha llamado «industria de la migración» se constituyeron en puentes entre los lugares de origen y destino. En este proceso, México se convirtió en un país de tránsito y los límites de la frontera sur se transformaron en espacios estratégicos.

En la migración de tránsito por México se definieron rutas de sur a norte, el tren de carga denominado La Bestia jugó un papel estratégico; también se han utilizado varios medios de transporte, desde autobuses, tractocamiones y vehículos diversos. Los polleros han sido parte de la «industria de la migración», quienes obtienen jugosas ganancias mediante un sistema de precios directamente proporcional a las medidas de contención; también los grupos delincuenciales que secuestran y extorsionan a los migrantes obtienen sumas millonarias. En suma, la migración se normalizó y se convirtió, parafraseando a David Harvey (2005), en fuente de «acumulación por desposesión». El uso político de la migración se hizo cada vez más recurrente: Barack Obama manipuló el voto latino en sus dos periodos, con la promesa de una reforma migratoria; Trump usó el tema migratorio en sentido inverso a Obama; mientras que Joe Biden prometió revertir las medidas antiinmigrantes de Trump y fue ampliamente favorecido por el voto latino.

El deterioro de las condiciones de vida de miles de centroamericanos y las medidas de control impuestas por México y Estados Unidos dieron lugar a la emergencia de las caravanas. El 20 de octubre de 2018, la frontera de Agua Caliente, entre Honduras y Guatemala, fue cerrada para evitar el paso de la primera caravana que partió de San Pedro Sula. Los migrantes lograron romper el cerco policial, las autoridades fueron rebasadas por la cantidad de migrantes que a su paso por toda la ruta iba aumentando hasta sumar más de 7 mil en territorio mexicano. Luego fueron llegando otras caravanas, la segunda con 3 mil y otras

${ }^{2}$ "La industria de la migración incluye agentes de migración, oficinas de viajes, banqueros, abogados, reclutadores de fuerza de trabajo, intérpretes y agentes de vivienda. Asimismo, puede incluir a miembros de una comunidad de migrantes como tenderos, sacerdotes y profesores, quienes ayudan a sus compatriotas de manera voluntaria o de tiempo parcial» (Castles, 2010:60). 
con menor número. Este fenómeno emergente desató la ira del presidente Donald Trump, quien envió tropas a su frontera sur para contener lo que denominó "ola invasiva» y obligó a México a adoptar medidas de contención con la ayuda de la Guardia Nacional y el Ejército.

A contracorriente del discurso antiinmigrante de Trump, Andrés Manuel López Obrador, todavía como presidente electo, adelantó la necesidad de formular un plan de desarrollo ${ }^{3}$ para convertir la migración en una opción voluntaria, no obligada por las circunstancias. Sin embargo, a pocas semanas de inicio de su gobierno comienza el giro en la política migratoria: el Instituto Nacional de Migración (INM) informó que el 28 de enero concluyó el programa emergente de emisión de Tarjetas de Visitante por Razones Humanitarias en la frontera con México. También indicó que hasta el 11 de febrero entregó 13 mil 270 Tarjetas de Visitante por Razones Humanitarias en Chiapas, Ciudad de México y Coahuila (INM, 2019).

La Patrulla Fronteriza reportó en el mes de mayo 144 mil 116 detenciones, el mayor número registrado desde el inicio del año 2019. Esto llevó a que el 30 del mismo mes el presidente Donald Trump comunicara la decisión de imponer aranceles progresivos a las importaciones, hasta 25 por ciento, de no implementarse medidas para contener la migración irregular:

On June 10th, the United States will impose a 5\% Tariff on all goods coming into our Country from Mexico, until such time as illegal migrants coming through Mexico, and into our Country. The Tariff will gradually increase until the Illegal Immigration problem is remedied (Tharoor, 6 de junio de 2019). [El 10 de junio, Estados Unidos impondrá un arancel de $5 \%$ a todos los bienes que ingresen a nuestro país desde México, hasta el momento en que los inmigrantes ilegales ingresen a México y lleguen a nuestro país. La tarifa aumentará gradualmente hasta que se solucione el problema de inmigración ilegal].

El comunicado de Trump llevó a que el presidente López Obrador enviara una misión a Washington. Finalmente, el 6 de junio México acordó implementar

${ }^{3}$ El estudio fue encargado a la Comisión Económica para América Latina y el Caribe (Cepal, 2019). El canciller Marcelo Ebrard presentó el 20 de mayo de 2020 el Plan de Desarrollo Integral para el Sur de México y Centroamérica. En esta oportunidad expresó: «El 1 de diciembre se firmó una declaración política, agradecemos a Cepal el tiempo récord en el que se hizo (...) esta es una hoja de ruta, de que tenemos que hacer para que cambie la realidad del sur de México, Guatemala, Honduras y El Salvador» (López, 2020). 
medidas de contención, ${ }^{4}$ los resultados pueden constatarse en una drástica disminución de las detenciones al finalizar junio, hasta llegar a poco más de 40 mil al final del año. La tendencia sigue bajando, en enero y febrero de 2020 la Patrulla Fronteriza reportó la detención de 36 mil 660 y 37 mil 199 migrantes, respectivamente.

Las fuertes presiones del gobierno estadounidense hicieron que la política migratoria se definiera por el lado de la contención. El 14 de junio de 2019, Tonatiuh Guillén, comisionado del INM, renuncia al cargo, lo que reafirma el giro en la política migratoria, y su manejo pasa de la Secretaría de Gobernación a la Secretaría de Relaciones Exteriores. De esta manera, México endureció su política migratoria, lo que terminó por restaurar y profundizar las medidas implementadas en el Programa Frontera Sur.

El gobierno de Estados Unidos manifestó su satisfacción por las acciones realizadas por México en materia de contención migratoria, aunque pidió mayores esfuerzos. Por otra parte, se obligó a los gobiernos de Guatemala, Honduras y El Salvador a firmar un pacto de «tercer país seguro». ${ }^{5}$ México ha tratado de convencer a Estados Unidos que la verdadera solución a la migración forzada es la puesta en marcha del Plan Integral para los países del Triángulo Norte; no obstante, hasta la conclusión del mandato del presidente Trump no se presentaron señales de apoyo a la propuesta del gobierno de la Cuarta Trasformación.

Mientras tanto, el escenario tendencial es la continuidad de la migración clandestina, irregular, y seguramente veremos crecer el número de solicitantes de refugio, una estrategia migratoria que ha dado resultados positivos porque la mayoría de las solicitudes de hondureños han sido aprobadas. ${ }^{6}$ El crecimiento de refugiados, aunque en términos del quantum general no es significativo, en los espacios de la frontera sur se expresa en diversas presiones de la sociedad local, que han llegado a enfrentamientos entre la policía local y migrantes que han ocupado espacios públicos para la economía informal.

${ }^{4}$ El 7 de junio se llega a un acuerdo entre ambos gobiernos, el comunicado conjunto señala: «México tomará medidas sin precedentes para aumentar la aplicación de la ley para frenar la migración irregular, para incluir el despliegue de su Guardia Nacional en todo México, dando prioridad a su frontera sur» (U.S. Department of State, 2019).

${ }^{5}$ El acuerdo con Guatemala fue firmado en la Casa Blanca el 26 de julio de 2019 entre Kevin McAleenan, secretario interino de Seguridad Nacional de los Estados Unidos, y Enrique Degenhart, ministro de Gobernación de Guatemala. Amnistía Internacional consideró que «cualquier intento de forzar a las familias e individuos que huyen de sus países de origen a buscar seguridad en Guatemala es indignante» (Orozco, 2019).

${ }^{6}$ Entre 2013 y 2019 el número de reconocidos positivos fue de 20 mil 866; y el número de reconocidos con protección complementaria ascendió a 5 mil 549. 
Durante el gobierno de Trump, que comprende los años fiscales 2017-2020, las detenciones efectuadas por la Patrulla Fronteriza sumaron 2 millones 372 mil 4, que frente a las 2 millones 56 mil 972 del último periodo de Obama (20132016) arroja una diferencia de 315 mil 232, una cifra nada despreciable si se toman en cuenta las restricciones impuestas por la covid-19. En la que se refiere a detención de menores de edad no acompañados del Triángulo Norte de Centroamérica, las cifras son muy similares: 147 mil 724 en la administración Trump, frente a 147 mil 790 con Obama. ${ }^{7}$

\section{Las últimas caravanas en la era de Trump}

El día 15 de enero de 2020 se formó una nueva caravana de migrantes con la mira de llegar a Estados Unidos. Un número aproximado de 400 personas cruzaron la frontera hondureña de Corinto, otro grupo más pequeño que venía atrás no pudo pasar y decidió esperar a que se reunieran más personas ( $\mathrm{La}$ Prensa, 15 de enero de 2020). Más tarde, entre el 15 y el 16, en el paso fronterizo guatemalteco de Agua Caliente (Esquipulas-Chiquimula), «pasaron mil 216 hondureños con documentación, y por el paso de El Cinchado (Puerto BarriosIzabal) otros 662, lo que hace un total de 2 mil 274 personas» (Henríquez, 2020). El jueves 16 por la tarde unos 200 migrantes se encontraban en Petén, mientras que otros 100 se hallaban en el paso fronterizo de Tecún Umán (Henríquez, 2020). En la mañana del viernes 17 los medios nacionales contabilizaron al menos 500 migrantes en esta frontera y en espera de la llegada de más personas (García, Domínguez y Coyoy, 2020). En total, la caravana se estimó en alrededor de 3 mil migrantes, menos de la mitad de la de octubre de 2018.

La reacción del gobierno de Honduras fue buscar culpables, Julián Pacheco, secretario de seguridad, declaró que hay personas que están financiando y promoviendo la caravana de migrantes. Indicó que la Policía Nacional está investigando y que en los próximos días presentarán un informe para desenmascarar a quienes promueven la caravana (La Prensa, 15 de enero de 2020).

De manera coincidente, el gobierno mexicano, a través de Alfonso Durazo, secretario de Seguridad y Protección Ciudadana, afirmó que detrás de las

${ }^{7}$ Cifras tomadas de la página oficial del Departamento de Seguridad Nacional (Department of Homeland Security), Oficina de Protección de Fronteras y Aduanas (CBP, 2020). https://www.cbp.gov/ newsroom/stats/sw-border-migration 
caravanas de migrantes hay intereses criminales por parte de los polleros. ${ }^{8}$ En el mismo sentido, la Secretaría de Gobernación declaró que colabora con las autoridades «sobre la investigación de más de 20 probables redes de traficantes de personas que operan en territorio mexicano con alcance internacional» (INM, 2020a).

El parte informativo del INM sobre la caravana de la esperanza fue el siguiente: «El Instituto Nacional de Migración (INM) informa que, del 18 al 27 de enero, en apego a la Ley de Migración y con pleno respeto a los derechos humanos, se realizó el retorno asistido de alrededor de 2 mil 303 personas migrantes hondureñas en 10 vuelos y 34 autobuses» (INM, 2020b).

En plena pandemia, en los primeros días de octubre se organizó la última caravana en la administración de Trump. En esta ocasión, el mensaje de las autoridades migratorias de México fue contundente: iquédense en casa! Tras romper el cerco militar, unos 3 mil migrantes hondureños ingresaron el jueves 1 de octubre a Guatemala (Garrán, 2020). Según la contabilidad del gobierno de Guatemala 3 mil 384 hondureños fueron obligados a regresar a su país debido al riesgo de la covid-19. El presidente Alejandro Giammattei refirió: «Respetamos el derecho a la migración siempre y cuando esta sea regulada y se cumpla con los requisitos y protocolos que han sido establecidos, y no como sucedió con este grupo de migrantes que irrumpió incluso con violencia en nuestro país» (La Jornada, 4 de octubre de 2020).

La crónica señala que «para viernes y sábado, cientos de policías y militares tendieron retenes en las carreteras obligando a retroceder a los migrantes, entre los que se contaban niños pequeños y personas en sillas de ruedas" (La Jornada, 4 de octubre de 2020). El gobierno de Guatemala mostró el rostro duro, intolerante, frente a un fenómeno que comparte: «Con los escudos antimotines al frente, los cascos recibiendo pedradas y las porras listas para utilizarse, comenzaron a caminar con cantos propios de la disciplina marcial y del cuerpo Kaibil como banda sonora. Acompasados por disparos de balas de salva» (Garrán, 2020).

A tono con la actitud del gobierno de Guatemala, México se pronunció por el no dejar pasar, con el mismo argumento de la pandemia. De esta manera, el "quédense en casa» resulta funcional a la política de contención ordenada por la Casa Blanca. En un comunicado fechado el 4 de octubre, el INM señala:

${ }^{8}$ Durazo afirmó: «No podemos ser ingenuos, tenemos una visión humanitaria respecto a la migración, pero en el caso de estas caravanas hay muchos intereses atrás de su organización» (Murillo, 2020). 
El Instituto Nacional de Migración (INM), dependiente de la Secretaría de Gobernación, las secretarías de Relaciones Exteriores (SRE), de la Defensa Nacional (Sedena), de Marina (Semar), así como la Guardia Nacional (GN), los gobiernos estatales de Chiapas y Tabasco, y la Embajada de Guatemala en México, coordinan acciones para garantizar un flujo migratorio seguro, ordenado y regular en la frontera sur del país (INM, 2020c, cursivas añadidas).

Contrario a lo que dice el INM, es evidente que no existe garantía del flujo migratorio, lo que refleja es más bien la sintonía de los gobiernos de Guatemala y México con el de Estados Unidos. La comunicación del INM es precisa: "Nuestro mensaje es muy claro para todos los que deseen migrar: iQuédense en casa! Es el momento de hacerlo en medio de la pandemia. Los gobiernos de Guatemala y de México trabajamos unidos, precisamente, para preservar sus vidas» (INM, 2020c).

La postura del gobierno reafirma el cambio en la política migratoria y «termina» una época en la que México se consideraba un país de tránsito migratorio hacia Estados Unidos, con todas las restricciones que implicaba en la práctica. ¿Cuáles son las consecuencias de esta política? Los efectos inmediatos son el encarecimiento de la migración clandestina, que a través del mecanismo de precios hace más selectiva la migración, y es más clara la división entre los que pueden y los que no pueden pagar un pollero.

El río Suchiate se convirtió en un referente geopolítico, del lado mexicano aguardan los agentes de migración y la Guardia Nacional; del lado guatemalteco está la esperanza, se planea el cruce de la frontera, se aguarda, se medita la estrategia, se acumulan fuerzas para pasar, ¿se ha sellado la frontera en el gobierno de la 4T?

\section{La paradoja del gobierno de la 4T: el sellamiento de la frontera sur de México}

Los postulados básicos del Plan Nacional de Desarrollo 2019-2024 (Secretaría de Hacienda y Crédito Público, 2019), en materia migratoria plantean:

a) A los extranjeros que llegan a nuestro territorio brindaremos respeto a sus derechos, hospitalidad y la posibilidad de que construyan aquí una nueva vida.

b) En lo que respecta al tratamiento de extranjeros migrantes en México - ya sea que se encuentren de paso hacia el país del norte o con propósitos de residencia 
en el territorio nacional-, la política del gobierno federal ha dado ya un giro en relación con la que había venido poniendo en práctica el régimen anterior.

c) El Ejecutivo Federal aplicará las medidas necesarias para garantizar que los extranjeros puedan transitar con seguridad por el territorio nacional o afincarse en él.

Estos postulados que serían parte del «nuevo paradigma» de la política migratoria pronto chocaron con la realidad. La aspiración de los gobiernos del Partido de Acción Nacional (PAN) y del Partido Revolucionario Institucional (PRI) de sellar la frontera sur de México, a instancias de la presión del gobierno de Estados Unidos, nunca se concretó a cabalidad. El que más se acercó fue el Programa Frontera Sur implementado en el verano de $2014,{ }^{9}$ con propósitos de contención y control de los flujos migratorios, con una visión de seguridad nacional. ${ }^{10}$

El Programa para la Seguridad Nacional 2014-2018, en el punto 4, «Fronteras, mares y flujos migratorios irregulares», enfatiza que los migrantes están expuestos a riesgos dado la «presencia de los grupos criminales que operan en las rutas migratorias que vinculan a Centroamérica con América del Norte». Destaca que «estos grupos delincuenciales aprovechan las condiciones de vulnerabilidad de los migrantes para realizar cobros de traslado, utilizarlos como portadores de enervantes o someterlos a la explotación sexual y laboral. En algunos casos, las organizaciones criminales también buscan reclutar a los migrantes para que ingresen a sus filas» (Diario Oficial de la Federación, 30 de abril de 2014).

Este fue el argumento que sirvió para afirmar la necesidad de "la consolidación de la Estrategia Integral de Atención a la Frontera Sur, (...) para fortalecer la presencia de la autoridad del Estado en la zona y coordinar acciones con los países centroamericanos para superar los desafíos comunes a la seguridad y al desarrollo, y avanzar en el establecimiento de una frontera más moderna, eficiente, próspera y segura» (DOF, 8 de julio de 2014, cursivas añadidas).

${ }^{9}$ El 8 de julio se crea por decreto presidencial la Coordinación para la Atención Integral de la Migración en la Frontera Sur (DOF, 8 de julio de 2014).

${ }^{10}$ En uno de los considerandos del decreto que crea la Coordinación para la Atención Integral de la Migración en la Frontera Sur es explícito al indicar "que el 30 de abril de 2014, se publicó en el Diario Oficial de la Federación el Programa para la Seguridad Nacional 2014-2018, en el que se establece que será fundamental la consolidación de la Estrategia Integral de Atención a la Frontera Sur, mecanismo impulsado por el Gobierno de la República desde junio de 2013 para fortalecer la presencia de la autoridad del Estado en la zona y coordinar acciones con los países centroamericanos para superar los desafíos comunes a la seguridad y al desarrollo, y avanzar en el establecimiento de una frontera más moderna, eficiente, próspera y segura» (DOF, 8 de julio de 2014). 
En la historia de la frontera sur y su relación con la migración se han registrado acciones que tienen como eje la seguridad, pero no para las personas, aunque ahora se trata de justificar la deportación con eufemismos como «retorno voluntario asistido», que de acuerdo con la Organización Internacional de la Migración (OIM) «es un componente indispensable del enfoque integral de la gestión de la migración». Este organismo considera que uno de sus principios fundacionales es «una migración ordenada, entre otras formas, mediante la asistencia para el retorno voluntario y la reintegración» (OIM, 2020).

Estamos lejos de los planteamientos de la OIM. Con la aplicación del Programa Frontera Sur más de 600 mil migrantes del Triángulo Norte fueron deportados. En el primer año de gobierno de la Cuarta Transformación $120 \mathrm{mil}$ 549 centroamericanos fueron deportados, de los cuales 98.5 por ciento correspondió a los países del norte de Centroamérica (Unidad de Política Migratoria, 2020).

El gobierno de la 4T se encuentra en medio de un serio dilema: entre cumplir con el canon de los derechos humanos y «dejar pasar» o «mandar obedeciendo» al gobierno de los Estados Unidos. En la práctica se ha inclinado por el «mandar obedeciendo». Entre el miedo y la esperanza los migrantes se aventuraron, de nuevo, a cruzar fronteras; sin embargo, en esta ocasión la frontera sur se convirtió en «el «muro» antes del muro» (Meyer e Isacson, 2019).

La libertad de tránsito y protección a los migrantes y sus familias es un recurso discursivo. Si emigrar es un derecho humano como proclama el artículo 13 de la Declaración Universal de los Derechos Humanos, y si México suscribió la Convención Internacional sobre la protección de los Derechos de todos los Trabajadores Migratorios y de sus familiares, estamos frente a una contradicción. Con justa razón De Sousa Santos refiere que «cuando la única libertad que le quede a esta mayoría sea la libertad de ser miserable, estaremos ante la miseria de la libertad. Para salir de este infierno, que parece programado por un plan voraz y poco inteligente, es necesario alterar la distribución desigual del miedo y la esperanza» (De Sousa, 26 de enero de 2020).

Los hechos definitorios de la politica antiinmigrante de Donald Trump

Durante su campaña política, Donald Trump utilizó el discurso antiinmigrante como uno de los ejes centrales para convencer a los electores estadounidenses. Con Trump se renueva la «economía del miedo» integrada por el complejo de 
empresas militares y securitarias (Davis, 2007). También se explota el miedo a los migrantes, a los indocumentados, a la gente de color, bajo el discurso del terrorismo, aderezado ahora con la frase del mandatario en turno "América primero", que envuelve una actitud ambivalente porque los migrantes contribuyen al proceso de acumulación.

El 20 de enero de 2017 Trump asumió la presidencia de Estados Unidos; el 25 ordenó la construcción de un muro en su frontera sur; también suscribió una orden ejecutiva para retirar fondos federales a las ciudades santuario (San Francisco, Chicago, Nueva York); el 27 decretó un veto migratorio con el propósito de suspender por 120 días la admisión de refugiados, al tiempo que negaba la entrada al país a ciudadanos de Siria, Irán, Sudán, Libia, Somalia, Yemen e Irak durante 90 días. Y así pasaron los días, en septiembre Jeff Sessions, fiscal general, anunció la derogación del Programa de Acción Diferida para los Llegados en la Infancia (DACA, por sus siglas en inglés).

El presupuesto para comenzar el muro fue aprobado en julio por la Cámara de Representantes, se trataba de mil 600 millones de dólares (El Economista, 27 de julio de 2017). Aunque no era la cantidad solicitada por el presidente Trump, se estaba cumpliendo con una de las promesas de campaña para «detener» la migración irregular. En el fondo es un doble juego: los «indeseados» son deseados por el capital agrícola, industrial y de servicios para tareas peligrosas, pesadas y sucias, pero se quiere de una fuerza de trabajo más controlada y regulada, por eso no es gratuito que el programa para migrantes temporales se haya disparado en los últimos meses. Por otra parte, la construcción del muro, así como el control de la frontera por medio de tecnología y equipamiento de la Patrulla Fronteriza son formas que adquiere la valorización del capital.

Es preciso producir mercancías que puedan ser vendidas, pero su naturaleza y destino importan poco al capital. La valorización del capital mediante la venta de materiales para las cámaras de gas de los campos de exterminio nazis sigue siendo el ejemplo más extremo de esta diferencia fundamental de capital con respecto a lo que se produce y vende siempre que exista una «demanda solvente». Pero la producción y la venta de armas de todo calibre traducen el mismo agnosticismo (Chesnais, 2007:3).

El 6 de febrero de 2018, el presidente Trump expresó: «Hemos identificado tres prioridades para crear un sistema de inmigración seguro, moderno y legal: 
asegurar la frontera, poner fin a la migración en cadena y cancelar la terrible lotería de visas» (The White House, 6 de febrero de 2018). Todo iba dirigido a construir un discurso justificatorio para la construcción del muro fronterizo.

En abril del mismo año, el gobierno de Washington había decidido enviar tropas a la frontera con México «ante el desgobierno en la zona y el aumento de inmigrantes indocumentados» (Agencias/La Jornada, 7 de abril de 2018). La idea era mandar entre 2 mil y 4 mil efectivos de la Guardia Nacional, «un despliegue de esta naturaleza es mayor que el que Estados Unidos mantiene en Siria y equivale a la mitad de las tropas que continúan en Irak» (Agencias/La Jornada, 7 de abril de 2018). Por su parte, el secretario de justicia Jeff Sessions ordenó tolerancia cero en contra de migrantes que crucen ilegalmente a Estados Unidos.

El 12 de julio de 2018, el presidente electo López Obrador envió una carta a Donald Trump donde esboza lo que sería su propuesta en materia migratoria $y$ de fronteras: «Propongo a usted, en primer lugar, hacer un esfuerzo por avanzar integralmente en las áreas sustantivas en las que está la esencia de la relación bilateral: el comercio, la migración, desarrollo y seguridad» (López Obrador, 2018). En la misiva, el presidente electo define las líneas generales de su proyecto: "El nuevo proyecto de nación que llevaremos a la práctica consistirá en desterrar la corrupción, abolir la impunidad, actuar con austeridad y destinar todo lo que se ahorre a financiar el desarrollo del país» (López Obrador, 2018, cursivas añadidas). Sobre la cuestión migratoria, la carta puntualiza:

Habrá muchos cambios, señor presidente Trump. Y en este nuevo ambiente de progreso con bienestar, estoy seguro que podremos llegar a acuerdos para enfrentar juntos tanto el fenómeno migratorio como el problema de la integridad fronteriza sobre la base de la cooperación para el desarrollo y bajo la premisa de que la paz y la tranquilidad son fruto de la justicia. Todo ello con absoluto respeto a los derechos humanos.

También, como se lo manifesté en nuestra conversación telefónica, considero que debe atenderse de manera integral y de fondo el problema migratorio, mediante un plan de desarrollo que incluya a los países centroamericanos donde millones de habitantes no tienen oportunidades de trabajo y se ven obligados a salir de sus pueblos para buscar la vida y mitigar su hambre y su pobreza (López Obrador, 2018). 
En agosto de 2018, todavía en proceso de formación del gabinete del presidente electo López Obrador, Alfonso Durazo anunció que se revisaría la cooperación en materia de seguridad con Estados Unidos, incluyendo la Iniciativa Mérida. Habló de la reorientación de esfuerzos hacia el impulso del desarrollo económico y social en el país, en el sur y en Centroamérica. Todavía no se habían presentado las caravanas de migrantes y el presidente electo adelantaba a funcionarios de Estados Unidos su propuesta de resolver el tráfico de drogas, armas y personas hacia Estados Unidos a través de fomentar el empleo y aumentar los niveles de vida en la frontera sur, incluyendo a los países de Centroamérica (Reuters/La Jornada, 4 de agosto de 2018).

En octubre del mismo año, en medio del avance de la primera caravana migrante, se lleva a cabo una conferencia de prensa conjunta con Kevin McAleenan, Comisionado de Aduanas y Protección Fronteriza de los Estados Unidos; el general de la Fuerza Aérea Terrence J. O'Shaughnessy, comandante del Comando de Defensa Aeroespacial de América del Norte y el Comando Norte de Estados Unidos, en la que expresaron:

A partir de esta tarde, seguimos rastreando a un gran grupo de aproximadamente 3 mil 500 que viajan a través del sur de México con la intención declarada de llegar a la frontera de Estados Unidos. Este grupo está cerca de la frontera entre Chiapas y Oaxaca, en el sur de México.

También conocemos un segundo gran grupo de migrantes en el cruce fronterizo de Ciudad Hidalgo entre Guatemala y México. Las estimaciones de tamaño para el segundo grupo son de alrededor de 3 mil y están formadas por unidades familiares y niños no acompañados que se han puesto en la mano de contrabandistas humanos violentos que pagan 7 mil por persona para hacer el viaje (U.S. Department of Defense, 2018).

El presidente Trump ordenó la «Operation Faithful Patriot» (Operación Patriota Fiel), que después cambiaría de nombre. Esta operación consistió en el envío de tropas a la frontera para detener a los migrantes que el presidente calificó de invasión, al respecto, el Pentágono refirió:

En términos de nuestros preparativos en la frontera, ayer lanzamos la Operación Línea Segura, una respuesta operativa flexible y de múltiples fases diseñada para garantizar que estamos preparados para cualquier cantidad de contingencias 
relacionadas con la llegada e intento de cruce de un gran grupo de migrantes en nuestra frontera, ya sea que intenten cruzar en un puerto de entrada o ilegalmente entre puertos de entrada (U.S. Department of Defense, 2018).

La operación es delirante, desproporcionada, pero con efecto mediático entre los estadounidenses, sobre todo en los grupos racistas y antiinmigrantes. El Pentágono habla de «dos docenas de activos aéreos para vigilancia y respuesta móvil, incluyendo cuatro helicópteros Blackhawks, seis helicópteros adicionales, así como múltiples activos de ala fija y sistemas de áreas no tripulados» (U.S. Department of Defense, 2018). Por si esto fuera poco, el Pentágono agrega:

Debido al gran tamaño de las posibles caravanas que pueden llegar a la frontera, el Departamento de Seguridad Nacional ha solicitado aún más el apoyo del Departamento de Defensa. Esto se suma a los 2 mil miembros del personal de la Guardia Nacional que ya están aumentando las operaciones bajo la Operación Guardian Support y están teniendo un impacto en la seguridad fronteriza, apoyando las detenciones y las incautaciones de drogas desde el año pasado (U.S. Department of Defense, 2018).

Como se puede leer, se trata de todo un operativo de guerra en contra de la «invasión». A este respecto, Noam Chomsky fue contundente al señalar: «Esta caravana de pobres y miserables huyen de la opresión severa, la violencia, el terror y la pobreza extrema». El filósofo recuerda que El Salvador, Guatemala y Honduras «han estado bajo la dura dominación de Estados Unidos desde hace mucho tiempo, particularmente desde la década de los 80». Además, consideró de «increíble farsa» el envío de tropas a la frontera sur de Estados Unidos, «los soldados enviados a la frontera superan en número a los niños que huyen", al tiempo que se desata una campaña para hacer creer a los estadounidenses que «estamos justo al borde de una invasión» (RT. Noticias internacionales, 3 de noviembre de 2018).

El 1 de diciembre de 2019 toma posesión el gobierno de Andrés Manuel López Obrador, al tiempo que las presiones del gobierno de Washington suben de nivel debido al aumento en la cantidad de migrantes que estaban llegando a la frontera sur de Estados Unidos. Entre diciembre y abril el gobierno de la $4 \mathrm{~T}$ recibió las más fuertes presiones del gobierno de Estados Unidos para endurecer las medidas antiinmigrantes, hasta la amenaza inminente de imponer impuestos al comercio de México con ese país. 
La presión de Estados Unidos en el giro de la política migratoria

El 26 de diciembre de 2019 el presidente López Obrador resumió la colaboración con Estados Unidos en el ámbito de la migración:

Se llegó a un acuerdo en muy buenos términos. Se alejó esa posibilidad de guerra comercial, que no hubiese sido buena para ninguna de las partes, no nos hubiese beneficiado a nadie. Se tomaron medidas y hay muy buenos resultados porque se contuvo el flujo migratorio de sur a norte. Se hizo dando opciones de trabajo a migrantes, protegiendo a niños, mujeres y sin violar derechos humanos (Presidencia de la República, 2019, cursiva añadidas).

El canciller Marcelo Ebrard presentó los resultados del «Plan de Migración y Desarrollo", así llamado el acuerdo con Estados Unidos para contener la migración centroamericana: «En síntesis, la Guardia Nacional rescató 58 mil 909, la Secretaría de Seguridad 41 mil 649 y el Instituto Nacional de Migración 178 mil 437. El total de migrantes rescatados este año: 278 mil 995» (Presidencia de la República, 2019). Ebrard detalla el punto de partida, y la tendencia que siguió el Plan entre mayo y diciembre de 2019:

El punto más alto, 144 mil 116, se alcanzó en el mes de mayo. Este es el reporte de todos los días de aprehensiones por parte de las autoridades norteamericanas en su territorio, es decir, en la frontera sur de Estados Unidos, norte de México. Ese fue el punto de partida.

Cada mes se fue reduciendo: primero 28 por ciento, luego 22, luego 23, luego 16, 14 y finalmente hasta llegar al mes de noviembre, para pasar de 144 mil 166 a 42 mil 710 en el mes de noviembre, con lo que sería la reducción más importante en estos flujos en las últimas décadas (Presidencia de la República, 2019).

Aquí es interesante destacar la estrategia utilizada para el logro de las cifras anteriores, en este aspecto el canciller refiere:

Se instalaron 21 puntos en la frontera sur y 20 en la frontera norte para hacer las revisiones correspondientes a cargo, en el sur, del general Vicente Hernández; y en el norte el general Lohmann. Quiero destacar que la Guardia Nacional, a pesar de haber tenido esta encomienda con esos números, no tiene ninguna 
recomendación de la Comisión Nacional de Derechos Humanos, lo cual es un mérito mayor.

En el caso de los sistemas vehiculares o aéreos de transporte también se hicieron las revisiones que aquí se informan: 41 mil 649 migrantes fueron rescatados en omnibuses o autobuses, 3 mil 479 autobuses. Y aquí destaco el problema serio de los tractocamiones. Fueron rescatados 2 mil 802 migrantes en 63 tractocamiones (Presidencia de la República, 2019).

Estas son las cuentas que el gobierno de la 4T rindió a Estados Unidos al final de 2019. Resultados que se traducen en la percepción de que México se puso al servicio del gobierno de la Casa Blanca. ¿Por qué cedió?, la razón fundamental fue la amenaza inminente de imponer aranceles progresivos al comercio de México con Estados Unidos, de 5 hasta 25 por ciento, lo que hubiera resultado, evidentemente, en pérdidas de miles de millones de dólares a la economía mexicana.

Después de la larga espera, desde el día de las votaciones, el 3 de noviembre, el sábado 7 por la tarde-noche, el candidato Joe Biden se declaró triunfador. En su discurso transmitido por televisión refirió que «la gente de esta nación ha hablado. Nos han entregado una victoria clara, una victoria convincente», subrayó que ganó las elecciones con 74 millones de votos, lo que representa «la mayor cantidad» de votos jamás obtenidos por un candidato en la historia del país (RT. Noticias, 8 de noviembre 2020).

Se necesitaban 270 votos para ganar la elección y las proyecciones de los medios CNN y NBC le atribuían en ese momento 273 votos electorales a Biden, en tanto que AP y Fox News proyectaron 290 votos. Trump indicó que había ganado la elección: «A los observadores no se les permitió estar en las salas de conteo. Gané las elecciones, obtuve 71 millones de votos legales. Sucedieron cosas malas que a nuestros observadores no se les permitió ver. Esto nunca había ocurrido antes» (RT. Noticias, 7 de noviembre de 2020).

El voto afroamericano y latino fue clave, según las encuestas se inclinó por el candidato demócrata en una proporción de 90 y 63 por ciento, respectivamente. Esto en el contexto de una política de odio racial que levantó una ola 
de manifestaciones encabezadas por el movimiento Black Lives Matter, y el acoso antiinmigrante. California, Georgia, Virginia, Nueva York, Pensilvania fueron clave para el triunfo de Biden. En Arizona los migrantes han sido asediados, debemos recordar la aprobación hace 10 años de la ley antiinmigrante SB1070 ejecutada por el alguacil Joe Arpaio. Aquí «los latinos aumentaron su participación en la votación de 16\% en 2016 a 18\% en 2020, prefiriendo a Biden por un margen de 59\%-39\%» (Adams, 2020).

El voto migrante para inclinar la balanza hacia uno u otro candidato ha sido objeto de cálculo político en los dos procesos electorales de Barack Obama, en las campañas de Donald Trump, particularmente en la primera, donde reiteradamente se refirió a los migrantes como escoria humana, y convirtió en fetiche la construcción del muro fronterizo; ahora Joe Biden, el candidato por el Partido Demócrata propone una línea que hace la diferencia con la política de Trump, ¿en qué consiste la diferencia entre la política migratoria de Trump y la prometida de Biden?

Como ofrecimiento de campaña, Biden planteó que, de ganar la elección, enviaría al Congreso un proyecto de ley para modernizar el «fracturado» sistema migratorio actual: «Desde el primer día promulgando los cambios necesarios para ayudar a la comunidad latina a prosperar»; la iniciativa de ley dará «una hoja de ruta hacia la ciudadanía» a los millones de inmigrantes indocumentados (Uno TV, 5 de agosto de 2020). Se refirió a la cancelación de la construcción del muro fronterizo, al restablecimiento del DACA, así como a la protección respecto a la separación de las familias aprehendidas por la Patrulla Fronteriza. En este sentido señaló que «una cuarta parte de todos los niños en Estados Unidos son latinos. Nuestro éxito colectivo depende de la comunidad latina» (Uno TV, 5 de agosto 2020).

¿Biden corresponderá a la población migrante? Es importante recordar que él formó parte de la política antiinmigrante de Barack Obama, que instituyó las «comunidades seguras», aumentó el poder de la Patrulla Fronteriza, reforzó la frontera y deportó el mayor número de centroamericanos. Por otra parte, hay que reconocer que Trump deja una herencia pesada con su política de tolerancia cero. Esta es la expresión más acabada del racismo, la xenofobia y la hipocresía en un país donde buena parte de la acumulación de capital recae sobre la fuerza laboral migrante. Tolerancia cero, aunque se ejemplifica con la separación de menores de sus padres, en realidad encierra el conjunto de políticas, expresadas en diversas iniciativas. 
En efecto, Trump fue enfático en indicar que la directiva de «tolerancia cero» se sostendría: "Mantendremos una frontera poderosa y continúa la tolerancia cero (...) Tenemos tolerancia cero para las personas que ingresan ilegalmente a nuestro país (...) La frontera sigue igual de difícil, pero queremos mantener unidas a las familias» (Haberman y Shear, 2018, cursivas añadidas). El abogado Peter Schey, del Centro por los Derechos Humanos y la Ley Constitucional, quien lleva el caso Flores, ${ }^{11}$ calificó la medida de tolerancia cero como un «martillazo que cae sobre la cabeza de padres e hijos» (Marrero, 2018).

La Oficina de Detención y Deportación (ICE, por sus siglas en inglés) refleja con claridad la política antiinmigrante de Trump, donde se evidencia la violación de los derechos humanos. Un informe elaborado por el Congreso revela el maltrato y la mala operación de los centros de detención: «Desde 2017, la Administración Trump ha otorgado a sus dos mayores contratistas de detención, CoreCivic y GEO Group, más de 5 mil millones de dólares en contratos para operar instalaciones de detención privadas» (Maloney y Raskin, 2020:3).

Más de 80 por ciento de las personas detenidas por ICE se encuentran en las instalaciones operadas por las dos contratistas mencionadas, donde de acuerdo con este informe se han encontrado "graves deficiencias médicas», así como negligencia y maltrato que «llevaron a la muerte de múltiples detenidos» (Maloney y Raskin, 2020:3).

La pandemia ha venido a constituirse en un factor clave que ha atenuado los flujos migratorios, constituyéndose en una «nueva frontera». ${ }^{12}$ La covid-19 ha sido un aliado a la política antiinmigrante de Trump, la pregunta es si ésta seguirá pesando en la política de Biden. Nuestra hipótesis es que habrá una reconsideración en dos aspectos sensibles: DACA y Estatus de Protección Temporal (TPS, por

${ }^{11}$ Con la aplicación de la política de tolerancia cero, el gobierno de Trump viola el llamado Acuerdo Flores, vigente desde 1997 y producto de 10 años de juicios contra políticas de detenciones de migrantes menores, mediante el cual se establecieron estándares mínimos, entre otros, "que los menores sean liberados sin retraso innecesario a un padre, guardián legal, familiar adulto, individuo designado por el padre, un programa de bienestar infantil; cuando un menor no puede ser liberado porque hay peligro de escape o riesgo a la seguridad pública, puede ser entonces detenido en un contexto lo menos restrictivo posible y de acuerdo a su edad y necesidades especiales. Se trata generalmente de un tipo de refugio, con licencia para el bienestar infantil y de baja seguridad; el acuerdo hace prioritaria la liberación del niño, excepto en dos casos: cuando se requiere para asegurar su presencia en la corte migratoria y cuando se requiere para garantizar la seguridad del menor y de los demás» (Marrero, 2018).

${ }^{12}$ Entre enero y septiembre de 2020, las deportaciones de la región Triángulo Norte de Centroamérica sumaron 29 mil 53, mientras que en el mismo periodo del año anterior alcanzó 119 mil 500; lo que significa una reducción 75.7 por ciento (Unidad de Política Migratoria, 2020). 
sus siglas en inglés). Ambos programas son convenientes para Estados Unidos, aportan al país y a la valorización del capital.

La covid-19 será usada para mantener la seguridad fronteriza, que es un componente de su política de seguridad nacional, inamovible. Puede abrirse la política de refugio y es de esperarse cierta flexibilidad a la migración irregular centroamericana y mexicana. Es posible que el escenario para México se mantenga, que el gobierno de la $4 \mathrm{~T}$ tenga que seguir con la política de contención. México, si quiere avanzar hacia un nuevo proyecto de nación, tendrá que poner todas sus cartas para obtener el apoyo de Estados Unidos, para llevar a cabo su Plan de Desarrollo Integral que involucra a los tres países del Triángulo Norte centroamericano.

Es importante recordar que Estados Unidos es el país más golpeado del mundo por la covid-19, la noticia del triunfo de Biden se acompañó con el registro de 10 millones de casos confirmados y 237 mil 564 fallecidos, de manera que un factor que pautará la política migratoria será la pandemia; según las primeras declaraciones del demócrata, "afrontamos un invierno muy obscuro», en el que "se podrían perder otras 200 mil vidas antes de que la cura esté ampliamente disponible» (Sin Embargo, 9 de noviembre de 2020).

Después de todo, «la afluencia de inmigrantes despierta la xenofobia de los países ricos de acogida y, en las congestionadas megalópolis, los exiliados de la miseria cavan sus propias zanjas» (Debray, 2016:81). No hay que olvidar que Trump obtuvo, según cifras preliminares, 70 millones 804 mil 457 votos, que lo coloca muy cerca de Biden con casi 75.2 millones. Jorge Zepeda se pregunta «¿cómo es posible que 70 millones de ciudadanos, prácticamente la mitad, votaron esta semana para mantener en el poder a un hombre que se convirtió en epíteto de la mentira, el bullying, el odio, la ignorancia, el narcisismo, el autoritarismo?» (Zepeda, 8 de noviembre de 2020), se responde expresando que «es reflejo del ascenso de estos atributos (...) en la conversión pública, en los usos y costumbres de hoy» (Zepeda, 8 de noviembre de 2020).

\section{A manera de conclusión}

¿Cuál es el escenario que se avecina después del triunfo de Joe Biden? La relación de vecindad de México con el país más poderoso del planeta define muchas cosas, la asimetría de poder es abismal y requiere un manejo diplomático 
extremadamente cuidadoso. Cuando decimos que "México está haciendo el trabajo sucio a Estados Unidos», se deben considerar varios factores. La disyuntiva entre "cooperar» en el gobierno de la Casa Blanca o no hacerlo tiene consecuencias económicas y políticas para el país.

En ese contexto, el escenario para México en lo que respecta a la movilidad humana es considerablemente complejo porque atiende a los intereses y presiones de Estados Unidos. El gobierno de la Cuarta Transformación tiene claro que la salida a la migración irregular está en resolver los problemas estructurales de Honduras, El Salvador y Guatemala, que son la fuente de las expulsiones. Desde principios de 2019, México propuso el Plan de Desarrollo Integral El SalvadorGuatemala-Honduras-México (Cepal, 2019), hasta ahora se mantiene en la congeladora porque requiere un acuerdo con Estados Unidos y los países centroamericanos, además de contar con el financiamiento que inicialmente se estimó entre 30 y 35 mil millones de dólares para cinco años, que ahora con la covid-19 resultará complicado disponer.

El escenario visible es que seguirá el éxodo centroamericano, con o sin caravanas, ahora con el ingrediente de la covid-19 que ha marcado de manera más clara y profunda las fronteras, que en el caso de la frontera sur de México se convierte en un nuevo muro. El éxodo se profundizará en los próximos años de la Cuarta Transformación, no sólo por las secuelas de la pandemia en materia de desempleo, pobreza, violencia, sino también por la ausencia de medidas de los gobiernos de la región para enfrentar la grave crisis económica y las vulnerabilidades de la región frente a la emergencia de fenómenos hidrometereológicos que han afectado gravemente a gran cantidad de población.

Por ahora México está solo, los países del norte de Centroamérica no tienen interés en hacer reformas profundas para evitar la migración forzada. Sus economías tienen una fuerte dependencia de las remesas: en 2019 Guatemala ingresó 10 mil 508.3 millones de dólares; ${ }^{13}$ El Salvador 5 mil 650.3 millones, ${ }^{14}$ y Honduras 5 mil 523.6 millones de dólares. ${ }^{15}$ Las remesas constituyen la principal fuente de divisas para los tres países; sin embargo, el costo humano del dinero es muy alto, no sólo por el número de desaparecidos y muertos.

El escenario es incierto, Trump ha perdido la presidencia, pero la pesadilla no ha terminado. El trumpismo estará presente en ambas Cámaras, pero sobre

\footnotetext{
${ }^{13}$ Véase Banco de Guatemala, 2020.

${ }^{14}$ Véase Banco Central de Reserva de El Salvador, 2020.

${ }^{15}$ Banco Central de Honduras, 2020.
} 
todo en la sociedad que acusa una profunda división, que se refleja nítidamente en la proporción de votos obtenidos por el republicano y en familias divididas: «Trump es como el catalizador de un terremoto que acaba de dividir dos continentes de pensamiento. Una vez que la Tierra se divide así, no hay vuelta atrás» (La Jornada, 7 de noviembre de 2020).

\section{Referencias}

Adams, David (2020), «El voto latino fue clave en la derrota de Biden, pero en Arizona la historia es otra», Univisión, 7 de noviembre, en https://www.univision.com/ noticias/elecciones-en-eeuu-2020/voto-latino-elecciones-presidenciales-bidentrump-arizona-florida

Agencias/La Jornada (7 de abril de 2018), "Acuerda Washington comenzar el despliegue de tropas en la frontera».

Banco Central de Honduras (2020), «Resultados encuesta semestral de remesas familiares 2020», en https://www.bch.hn/download/remesas_familiares/remesas_ familiares_012020.pdf

Banco Central de Reserva de El Salvador (2020), «Ingresos mensuales de remesas familiares», en https://www.bcr.gob.sv/bcrsite/?x21=46

Banco de Guatemala (2020), "Ingreso de divisas por remesas familiares», en http:// www.banguat.gob.gt/inc/ver.asp?id=/estaeco/remesas/remfam2010_2019. htm\&e $=143294$

Castles, Stephen y Mark J. Miller (2004), La era de la migración. Movimientos internacionales de población en el mundo moderno, México, Universidad Autónoma de Zacatecas/Instituto Nacional de Migración/Miguel Ángel Porrúa.

Castles, Stephen (2010), «Migración irregular: causas, tipos y dimensiones regionales», Migración y Desarrollo, 7(15), pp. 49-80.

Chesnais, François (1 de abril de 2007), «Mundialización: extrema pobreza, destrucción del medio ambiente y guerras... La irracionalidad fundamental del capitalismo está en el núcleo de la crisis de civilización planetaria», Agenpress.

Comisión Económica para América Latina y el Caribe (Cepal) (2019), Plan de Desarrollo Integral El Salvador-Guatemala-Honduras-México, México, Naciones Unidas.

Customs and Border Protection (СBP) (2020), «Southwest Border Migration FY 2020», en https://www.cbp.gov/newsroom/stats/sw-border-migration

Davis, Mike (2007), Ciudades muertas, Madrid, Traficantes de Sueños. 
De Sousa Santos, Boaventura (26 de enero de 2020), «Para una nueva declaración universal de los derechos humanos", La Jornada.

Debray, Régis (2016), Elogio de las fronteras, Barcelona, Gedisa.

Diario Oficial de la Federación (DOF) (8 de julio de 2014), «Decreto por el que se crea la Coordinación para la Atención Integral de la Migración en la Frontera Sur», México, Secretaría de Gobernación.

(30 de abril de 2014), «Programa para la Seguridad Nacional 2014-2018. Una política multidimensional para México en el siglo XXI», México, Secretaría de Gobernación.

El Economista (27 de julio de 2017), «Aprueban 1,600 mdd para empezar a construir el muro», en http://eleconomista.com.mx/internacional/2017/07/27/aprueban-1600 -mdd-iniciar-construccion-muro

García, Oscar, Andrea Domínguez y Alex Coyoy (2020), «Migrantes hondureños 1legan a Tecún Umán y advierten que pasarán en grupo hacia México», Prensa Libre, en https://www.prensalibre.com/guatemala/migrantes/migrantes-hondurenos -llegan-a-tecun-uman-y-advierten-que-pasaran-en-grupo-hacia-mexico/

Garrán, Felipe (4 de octubre de 2020), «¿Sabes qué me hicieron?: la crónica del cruento choque de migrantes hondureños y el Ejército que mató el sueño americano en Guatemala», Prensa Libre, en https://www.prensalibre.com/guatemala/migrantes/ piedras-y-bombas-molotov-la-cronica-del-cruento-choque-de-migrantes-hondurenos -en-guatemala-que-mato-el-sueno-americano/

Gorz, André (2000), Miserias del presente, riqueza de lo posible, Buenos Aires, Paidós.

Haberman, Maggie y Michael D. Shear (20 de junio de 2018), «Trump ordena detener juntos a padres e hijos y acaba con las separaciones familiares», The New York Times, en https://www.nytimes.com/es/2018/06/20/espanol/trump-separacionpadres-hijos-frontera.html

Harvey, David (2005), «El «nuevos imperialismo: acumulación por desposesión», Socialist Register 2004, Buenos Aires, Clacso, pp. 99-129.

Henríquez, Elio (7 de enero de 2020), "Avanza en Guatemala una caravana de 3 mil migrantes hondureños», La Jornada.

INM (12 de febrero de 2019), "Finaliza programa emergente de emisión de Tarjetas de Visitante por Razones Humanitarias», en https://www.gob.mx/inm/articulos/ finaliza-programa-emergente-de-emision-de-tarjetas-de-visitante-por-razoneshumanitarias?idiom $=$ es

(26 de enero 2020a), "Colabora INM en investigación de más de 20 probables redes de traficantes de personas en México», Boletín núm. 027/2020, en 
https://www.gob.mx/inm/prensa/colabora-inm-en-investigacion-de-mas-de20-probables -redes-de-traficantes-de-personas-en-mexico-233008

(27 de enero 2020b), "Realiza INM retorno asistido de más de 2 mil personas migrantes hondureñas», Boletín núm. 029/2020, en https://www.gob.mx/ $\mathrm{inm} /$ prensa/realiza-inm-retorno-asistido-de-mas-de-2-mil-personas-migrantes -hondurenas-233137

(4 de noviembre de 2020c), «Supervisan operativo de 24 horas del río Suchiate, Chiapas, a El Ceibo, Tabasco, INM, SER, Sedena, Semar, GN, Embajada de Guatemala y Salud», Boletín núm. 373/2020, en https:/www.gob.mx/inm/ prensa/supervisan-operativo-de-24-hrs-del-rio-suchiate-chiapas-a-el-ceibo -tabasco-inm-sre-sedena-semar-gn-embajada-de-guatemala-y-salud

La Jornada (4 de octubre de 2020), "Guatemala se convierte en muro infranqueable para caravana migrante», en https://www.jornada.com.mx/sin-fronteras/2020/10/04/ guatemala-se-convierte-en-muro-infranqueable-para-caravana-migrante-2813. html

(7 de noviembre de 2020), "Migrantes ante el incierto futuro post Trump», en https://www.jornada.com.mx/sin-fronteras/2020/11/07/migrantesante-el-incierto-futuro-post-trump-4612.html

La Prensa (15 de enero de 2020), «Hondureños que lleguen a México sin visa serán deportados», en https://www.laprensa.hn/premium/1349281-410/hondurenos -lleguen-mexico-sin-visa-seran-deportados-caravana-migrante

López Obrador, Andrés Manuel (22 de julio de 2018), «Carta completa que AMLO envió a Trump con plan bilateral», La Jornada, en http://www.jornada.com.mx/ ultimas/2018/07/22/carta-completa-que-amlo-envio-a-trump-con-plan-bilateral $-4891 . h t m 1$

López, Jannet (20 de mayo 2020), "México presenta proyecto de desarrollo para la frontera sur», Milenio, en https://www.milenio.com/politica/mexico-presenta -plan-orientar-politica-migratoria-frontera-sur

Maloney, Carolyn B. y Raskin Jamie (septiembre de 2020), "The Trump administration's mistreatment of detained immigrants: deaths and deficient medical care by For-Profit detention Contractors», Staff Report Committee on Oversight and Reform and Subcommittee on Civil Rights and Civil Liberties U.S. House of Representatives.

Marrero, Pilar (2018), «Abogado principal de caso Flores alega que separación familiar estaría violando el acuerdo", en https:/eldiariony.com/2018/06/18/abogado 
-principal-de-caso-flores-alega-que-separacion-familiar-estaria-violando-elacuerdo/

Meyer, Maureen y Adam Isacson (2019), "El «muro» antes del muro. El aumento de medidas contra la migración en la frontera sur de México», Washington, Wola, en https://www.wola.org/wp-content/uploads/2019/12/SPN-EXEC-SUMMARY2019-Southern-Border-Report.pdf

Murillo, Eduardo (22 de enero de 2020), "Intereses criminales detrás de caravanas migrantes: Durazo", La Jornada, en https://www.jornada.com.mx/ultimas/politica /2020/01/22/intereses-criminales-detras-de-caravanas-migrantes-durazo-4863. html

OIM (2020), «Retorno voluntario asistido y reintegración», en https://www.iom.int/es/ retorno-voluntario-asistido-y-reintegracion

Orozco, Andrea (27 de julio de 2019), "Acuerdo migratorio entre Guatemala y EE.UU. es indignante), dice Amnistía Internacional», Prensa Libre, en https://www. prensalibre.com/guatemala/politica/acuerdo-migratorio-entre-guatemala-y-ee -uu-es-indignante-dice-amnistia-internacional/

Presidencia de la República (26 de diciembre de 2019), «Diálogo con medios de comunicación encabezado por el presidente Andrés Manuel López Obrador desde Palacio Nacional», Versión estenográfica, Palacio Nacional, en https://www.jornada. com.mx/ultimas/mundo/2020/03/11/trump-mantendra-programa-permaneceren-mexico-9605.html

Reuters/La Jornada (4 de agosto de 2018), "A evaluación, la cooperación en seguridad con EU, dice Durazo».

RT. Noticias (7 de noviembre de 2020), «Trump asegura que ganó las elecciones con $>71$ millones de votos legales` y denuncia que a los observadores «no se les permitiós asistir al recuento», en https://actualidad.rt.com/actualidad/372751-trump -asegura-ganar-elecciones-71-millones-votos

(8 de noviembre de 2020), «Joe Biden proclama su «victoria convincente» en las presidenciales y llama a EE.UU. ‘faro para el mundo», en https://actualidad. rt.com/actualidad/372765-biden-dirige-nacion-proyectado-ganador-elecciones (3 de noviembre de 2018), "Chomsky: «caravana huye de la miseria y de los horrores de los que EE. UU. es responsable», en https://actualidad.rt.com/ actualidad/294340-chomsky-caravana-migrantes-eeuu -responsable

Secretaría de Hacienda y Crédito Público (12 de julio de 2019), «Decreto por el que se aprueba el Plan Nacional de Desarrollo 2019-2024", Diario Oficial de la Federación. 
Sin Embargo (9 de noviembre de 2020), "Biden advierte que otros 200 mil morirán por covid en EU y que se viene un invierno muy oscuro», en https://www.sinembargo. $\mathrm{mx} / 09-11-2020 / 3890838$

Tharoor, Ishaan (6 de junio de 2019), «Trump blames Mexico for problem with no borders», The Washington Post, en https://www.washingtonpost.com/world /2019/06/07/trump-blames-mexico-problem-with-no-borders/?utm_term=. b09dc1e7070a

The White House ( 6 de febrero de 2018), «Remarks by president Trump at law enforcement roundtable on MS-13», en https://www.whitehouse.gov/briefingsstatements/remarks-president-trump-law-enforcement-roundtable-ms-13/

Trump, Donald (30 de mayo 2019), Twitter, en https:/twitter.com/realDonaldTrump/ status/1134240653926232064

U.S. Department of Defense (29 de octubre 2018), «Homeland Security and Defense Department officials joint press conference on the Defense Department Deployment to the southwest border», en https://www.defense.gov/Newsroom/Transcripts/ Transcript/Article/1675870/homeland-security-and-defense-department-officials -joint-press-conference-on-th/

U.S. Department of State (7 de junio de 2019), «U.S.-Mexico joint declaration», Washington, D.C., en https://www.state.gov/u-s-mexico-joint-declaration/

Unidad de Política Migratoria (2020), «Boletines estadísticos», en http://www.politica migratoria.gob.mx/es/PoliticaMigratoria/Boletines_Estadisticos

Uno TV (5 de agosto de 2020), "Joe Biden promete ponerle fin al proyecto del muro de Trump», en https://www.unotv.com/internacional/joe-biden-promete-ponerle -fin-al-proyecto-del-muro-de-trump/

Zepeda, Jorge (8 de noviembre de 2020), "Trump se va, se queda lo que trajo», Sin Embargo, en https://www.sinembargo.mx/08-11-2020/3890096 